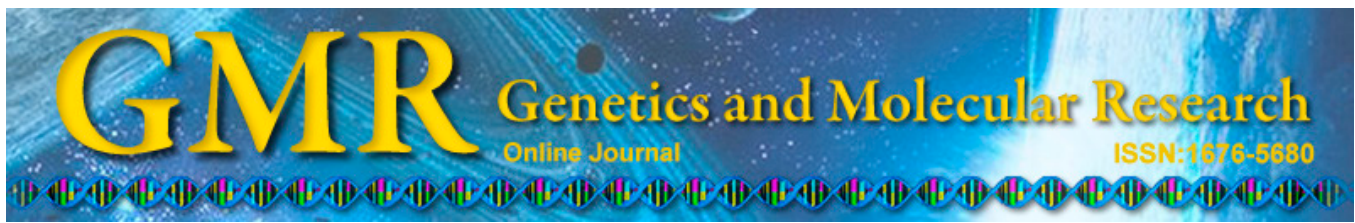

Short Communication

\title{
Isolation and characterization of microsatellite loci in the purpleback flying squid (Sthenoteuthis oualaniensis)
}

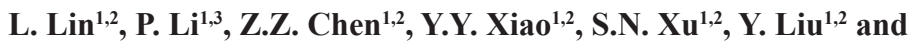 \\ C.H. $\mathrm{Li}^{1,2}$
}

${ }^{1}$ Key Laboratory for Exploitation and Utilization of Marine Fisheries Resources in the South China Sea, Ministry of Agriculture, Guangzhou, China ${ }^{2}$ South China Sea Fisheries Research Institute, Chinese Academy of Fishery Sciences, Guangzhou, China

${ }^{3}$ School of Fisheries and Life Science, Shanghai Ocean University, Shanghai, China

Corresponding author: C.H. Li

E-mail: scslch@vip.163.com

Genet. Mol. Res. 14 (3): 7630-7633 (2015)

Received August 11, 2014

Accepted April 17, 2015

Published July 13, 2015

DOI http://dx.doi.org/10.4238/2015.July.13.7

\begin{abstract}
The purpleback flying squid (Sthenoteuthis oualaniensis) is a pelagic squid with tremendous potential for commercial exploitation. We isolated and characterized 21 polymorphic microsatellite loci for $S$. oualaniensis using a $(\mathrm{GT})_{13^{-}}{ }^{-}$ enriched genomic library. The number of alleles per locus varied from 6 to 32. The observed and expected heterozygosities ranged from 0.188 to 0.890 , and 0.537 to 0.968 , respectively. No significant linkage disequilibrium was detected at these loci. Five loci significantly deviated from the Hardy-Weinberg equilibrium, and four loci may have exhibited null alleles. These microsatellite markers will facilitate
\end{abstract}


further studies in population genetics and the sustainable utilization of $S$. oualaniensis.

Key words: Purpleback flying squid; Sthenoteuthis oualaniensis; Population genetics; Microsatellite

\section{INTRODUCTION}

The purpleback flying squid (Sthenoteuthis oualaniensis) is a pelagic squid that is widely distributed in the tropical and subtropical areas of the Pacific and the Indian Oceans, particularly in the South China Sea and the northwest areas of the Indian Ocean (Nesis, 1977; Mohamed et al., 2006). In recent years, because of the drastic decline in traditional fishery resources in the South China Sea, the focus of marine exploitation has shifted to S. oualaniensis owing to its high biomass, short life cycle, high growth rate, and high fecundity (Chen et al., 2007; Zhang et al., 2010). Analysis of the genetic diversity and population structure of $S$. oualaniensis can provide essential information for the better management and sustainable utilization of this species.

Microsatellites are one of the best markers for studying population genetics in pelagic marine organisms, because they are reproducible, multi-allelic, co-dominant, and relatively abundant (Zhan et al., 2009). No microsatellite marker is currently available for $S$. oualaniensis; therefore, we developed and characterized 21 microsatellite markers for this species. These markers will facilitate further studies in population genetics and the sustainable utilization of S. oualaniensis.

\section{MATERIAL AND METHODS}

A (GT) $)_{\mathrm{n}}$-enriched genomic library of $S$. oualaniensis was constructed following a previously published method (Ma and Chen, 2009). In brief, genomic DNA was extracted from muscle tissue and digested with the MseI restriction enzyme (New England Biolabs, USA) at $37^{\circ} \mathrm{C}$ for $3 \mathrm{~h}$. The digested fragments were ligated to specific adapters (5'-TACTCAGGAACTCAT-3'/5'-GACGATGAGTCCTGAG-3') using T4 DNA ligase (Fermentas, USA) at $16^{\circ} \mathrm{C}$ for more than $8 \mathrm{~h}$. The ligated fragments were amplified in a 25$\mu \mathrm{L}$ reaction system, using an adapter-specific primer (5'-GATGAGTCTGAGTAA-3'), to verify the ligation and increase the DNA concentration. The polymerase chain reaction (PCR) products were hybridized to biotin-labeled $(\mathrm{GT})_{13}$ probes, and the hybridized complexes were captured by streptavidin-coated magnetic beads (Promega, USA). Subsequently, nonspecific binding and unbound DNA was removed by several non-stringent and stringent washes. The microsatellite-enriched DNA fragments eluted from the magnetic beads were amplified using the adapter-specific primer, and the amplified products were purified on $1.5 \%$ agarose gel. The DNA fragments (ranging in size from 500 to $1000 \mathrm{bp}$ ) were extracted using a DNA gel extraction kit (Axygen, USA) and ligated into pMD18-T vectors (Takara, Japan), which were then transformed into Escherichia coli DH5a-competent cells. The positive clones were sequenced on an ABI 3730 automated DNA sequencer (Applied Biosystems, USA).

DNA sequences containing microsatellite loci were selected, and primers were designed based on the flanking regions of the microsatellite loci using the Primer Premier 5 software (Premier Biosoft International, USA). The designed primer pairs were evaluated us- 
ing 50 individuals of $S$. oualaniensis collected from the South China Sea. PCR amplifications were performed separately in a $25-\mu \mathrm{L}$ reaction volume that contained $0.4 \mu \mathrm{M}$ primer (each), $0.2 \mathrm{mM}$ dNTP (each), $2 \mathrm{mM} \mathrm{MgCl}$, 1 X PCR buffer, $1 \mathrm{U}$ Taq polymerase (Takara, Japan), and 50 to 100 ng template DNA. The PCR cycling profile was as follows: an initial denaturation at $94^{\circ} \mathrm{C}$ for $5 \mathrm{~min} ; 35$ cycles of $45 \mathrm{~s}$ at $94^{\circ} \mathrm{C}, 45 \mathrm{~s}$ at the optimal annealing temperature (Table 1), and $45 \mathrm{~s}$ at $72^{\circ} \mathrm{C}$, followed by a final extension step at $72^{\circ} \mathrm{C}$ for $10 \mathrm{~min}$. The PCR products were separated on $6 \%$ denaturing polyacrylamide gels, and visualized by silver staining. Allele size was estimated using a pBR322/MspI marker (TianGen, China).

The observed and expected heterozygosities, the Hardy-Weinberg equilibrium, and linkage disequilibrium were calculated using Genepop 4.0 (Rousset, 2007). Null allele frequencies were estimated using Micro Checker 2.2.3 (Van Oosterhout et al., 2004). Bonferroni corrections (Rice, 1989) were used to correct the results of all of the multiple tests conducted.

Table 1. Characterization of 21 polymorphic microsatellite loci for Sthenoteuthis oualaniensis.

\begin{tabular}{|c|c|c|c|c|c|c|c|c|}
\hline Locus & Repeat motif & Primer sequences $\left(5^{\prime}-3^{\prime}\right)$ & Size (bp) & $\mathrm{Ta}\left({ }^{\circ} \mathrm{C}\right)$ & $N_{\mathrm{A}}$ & $H_{\mathrm{O}}$ & $H_{\mathrm{E}}$ & $\begin{array}{c}\text { GenBank } \\
\text { accession No. }\end{array}$ \\
\hline $\mathrm{SO} 2$ & $(\mathrm{GT})_{6}$ & $\begin{array}{l}\text { F: CACAACTAATCAAGTGGACAA } \\
\text { R: TATGCTATCAATAAACCGAAA }\end{array}$ & $270-309$ & 58 & 8 & 0.440 & 0.537 & KF999033 \\
\hline SO6 & $(\mathrm{TG})_{10}(\mathrm{GT})_{6}(\mathrm{GT})_{16}$ & $\begin{array}{l}\text { F: TTTAGTTTATACCCTCAATGT } \\
\text { R: CACTCATACAGACATCCATAC }\end{array}$ & $250-331$ & 58 & 23 & 0.804 & 0.935 & KF999034 \\
\hline $\mathrm{SO}^{* \dagger}$ & $(\mathrm{CA})_{5}$ & $\begin{array}{l}\text { F: TCGTGGAAAATTGAAACA } \\
\text { R: AAAATGCCCTCATAAACA }\end{array}$ & $230-260$ & 58 & 22 & 0.740 & 0.897 & KF999035 \\
\hline SO14*† & $(\mathrm{AC})_{5}$ & $\begin{array}{l}\text { F: CACCAAAGTAACTGGGACAAC } \\
\text { R: AGTTTCATTACATGAAGCGTG }\end{array}$ & $195-258$ & 60 & 6 & 0.283 & 0.707 & KF999036 \\
\hline SO18 & $(\mathrm{TG})_{16}(\mathrm{GT})_{17}$ & $\begin{array}{l}\text { F: TCAAGCACCAAACGAGAT } \\
\text { R: AAATGAGCAAAACGAACA }\end{array}$ & $255-337$ & 60 & 18 & 0.890 & 0.900 & KF999037 \\
\hline $\mathrm{SO} 20$ & $(\mathrm{AC})_{8}$ & $\begin{array}{l}\text { F: CCCACCAGAAGTTTTAGG } \\
\text { R: AGTCCCAATCTCGTCTTT }\end{array}$ & $231-285$ & 60 & 13 & 0.760 & 0.882 & KF999038 \\
\hline SO23 & $(\mathrm{GT})_{29}$ & $\begin{array}{l}\text { F: GCCTTTGTATGACCAGAT } \\
\text { R: TGAGTCTACTTCAATGGGT }\end{array}$ & $287-349$ & 62 & 18 & 0.850 & 0.924 & KF999039 \\
\hline SO24 & $(\mathrm{CT})_{6}(\mathrm{CA})_{22}$ & $\begin{array}{l}\text { F: TGCTGAGTTTTCCAAAGG } \\
\text { R: ATATTCGTAGATGACACCATA }\end{array}$ & $315-388$ & 58 & 14 & 0.780 & 0.896 & KF999040 \\
\hline SO39 & $(\mathrm{TG})_{10}(\mathrm{GT})_{6}(\mathrm{GT})_{6}$ & $\begin{array}{l}\text { F: TTTTAGTTTATACCCTCAATG } \\
\text { R: CACTCATACAGACATCCATAC }\end{array}$ & $264-333$ & 58 & 16 & 0.826 & 0.933 & KF999041 \\
\hline SO45 & $(\mathrm{TCA})_{11}$ & $\begin{array}{l}\text { F : CATTCTACCTTTAGTCGC } \\
\text { R: GTGTCAAACAATGGATTT }\end{array}$ & $300-356$ & 58 & 17 & 0.847 & 0.911 & KF999042 \\
\hline $\mathrm{SO} 47^{* \dagger}$ & $(\mathrm{TG})_{25}$ & $\begin{array}{l}\text { F: ATTGATAGCCTTTGGTGA } \\
\text { R: TGAGCCTTATTTCGGTCT }\end{array}$ & $238-289$ & 60 & 20 & 0.256 & 0.940 & KF999043 \\
\hline SO51 & $(\mathrm{AC})_{15}(\mathrm{AC})_{11}(\mathrm{AG})_{5}$ & $\begin{array}{l}\text { F: ATCGCTGTAGGGTGAGGA } \\
\text { R: CACTATGCCAAATGTTGTTT }\end{array}$ & $286-355$ & 63 & 13 & 0.790 & 0.878 & KF999044 \\
\hline SO59 & $(\mathrm{AG})_{16}(\mathrm{GT})_{5}(\mathrm{TG})_{10}(\mathrm{GA})_{9}$ & $\begin{array}{l}\text { F: CCAAATACGCATTCAAACAA } \\
\text { R: GATCACAAAGCGAATCCTTC }\end{array}$ & $262-346$ & 63 & 23 & 0.871 & 0.935 & KF999045 \\
\hline SO60 & $(\mathrm{TG})_{20}$ & $\begin{array}{l}\text { F: TTTATCGGAAAATCGCTAC } \\
\text { R: TACGCAGAATCATACGCTA }\end{array}$ & $179-252$ & 60 & 18 & 0.890 & 0.920 & KF999046 \\
\hline SO74 & $(\mathrm{GT})_{9}$ & $\begin{array}{l}\text { F: AACGATTGGTCCTCAGTG } \\
\text { R: CCTTGCTCCTACAGTCTTA }\end{array}$ & $220-258$ & 63 & 11 & 0.776 & 0.781 & KF999047 \\
\hline $\mathrm{SO} 79^{* \dagger}$ & $(\mathrm{ATGT})_{6}$ & $\begin{array}{l}\text { F: TCACTATCGTTATAGACCCAG } \\
\text { R: TTTGAGATTTTGAGCGTTG }\end{array}$ & 268-309 & 63 & 13 & 0.188 & 0.871 & KF999048 \\
\hline SO80 & $(\mathrm{CA})_{17}$ & $\begin{array}{l}\text { F: GATTAGTGGACGTTTCCG } \\
\text { R: TCCCGATTCAATTCTTGT }\end{array}$ & $262-324$ & 60 & 15 & 0.833 & 0.916 & KF999049 \\
\hline SO83 & $(\mathrm{GA})_{8}(\mathrm{GA})_{20}$ & $\begin{array}{l}\text { F: GTACCCACAACCTCACAT } \\
\text { R: TTTTCCCAGCTATAAATT }\end{array}$ & $190-277$ & 50 & 32 & 0.812 & 0.968 & KF999050 \\
\hline SO86 & $(\mathrm{CA})_{7}$ & $\begin{array}{l}\text { F: TGGAAGGGGAGAAAAGTA } \\
\text { R: GATGGGAAATATGATGCA }\end{array}$ & $299-325$ & 60 & 6 & 0.360 & 0.322 & KF999051 \\
\hline SO96 & $(\mathrm{TG})_{29}$ & $\begin{array}{l}\text { F: CTCCAGTGCCACCCCAGTAAA } \\
\text { R: AGAGGACCATAACCCCGACAA }\end{array}$ & $220-272$ & 60 & 16 & 0.747 & 0.878 & KF999053 \\
\hline $\mathrm{SO} 101^{\dagger}$ & $(\mathrm{AC})_{11}(\mathrm{CA})_{5}$ & $\begin{array}{l}\text { F: ATGCCTGGAAGATGTGAATG } \\
\text { R: TTGGCTTTGCTGAACGAATA }\end{array}$ & $314-333$ & 58 & 13 & 0.490 & 0.879 & KF999055 \\
\hline
\end{tabular}

Ta $=$ optimized annealing temperature; $N_{\mathrm{A}}=$ number of alleles; $H_{\mathrm{O}}=$ observed heterozygosity; $H_{\mathrm{E}}=$ expected heterozygosity; *locus may harbor null alleles (estimated null allele frequency $>5 \%$ ); ${ }^{\dagger}$ locus deviated from HardyWeinberg proportions (adjusted $\mathrm{P}<0.0023$ ). 


\section{RESULTS AND DISCUSSION}

In total, 150 positive clones were identified and sequenced, and microsatellite repeats were found in 106 sequences. Fifty-three primer pairs were designed based on the microsatellite sequences. Twenty-one loci were amplified cleanly and found to be polymorphic, and the number of alleles per locus varied from 6 to 32. The observed and expected heterozygosities ranged from 0.188 to 0.890 , and 0.537 to 0.968 , respectively. No significant linkage disequilibrium was detected among these loci. Five loci deviated significantly from the Hardy-Weinberg equilibrium after a Bonferroni correction (adjusted $\mathrm{P}<0.0023$ ), which may have been due to the small sample size or the existence of null alleles. Null alleles may have been present at four loci (estimated null allele frequency $>5 \%$ ). These markers will serve as useful tools for population genetics and sustainable utilization studies of S. oualaniensis (Table 1).

\section{ACKNOWLEDGMENTS}

Research supported by the Special Fund for Agro-Scientific Research in the Public Interest (Grant \#201403008), the China Postdoctoral Science Foundation (Grant \#2013M531882), and the Planned Science and Technology Project of Guangdong Province (Grant \#2011B031100001).

\section{REFERENCES}

Chen XJ, Liu BL, Tian SQ, Qian WG, et al. (2007). Fishery biology of purpleback squid, Sthenoteuthis oualaniensis, in the northwest Indian Ocean. Fish. Res. 83: 98-104.

Ma HY and Chen SL (2009). Isolation and characterization of 31 polymorphic microsatellite markers in: barfin flounder (Verasper moseri) and the cross-species amplification in spotted halibut (Verasper moseri). Conserv. Genet. 10: 1591-1595.

Mohamed KS, Joseph M and Alloycious PS (2006). Population characteristics and some aspects of the biology of oceanic squid Sthenoteuthis oualaniensis (Lesson, 1830). J. Mar. Biol. Ass. 48: 256-259.

Nesis KN (1977). Population structure in the squid Sthenoteuthis oualaniensis (Lesson 1930) in the Western Tropical Pacific. Oceanology 107: 15-29.

Rice WR (1989). Analyzing tables of statistical tests. Evolution 43: 223-225.

Rousset F (2007). GENEPOP'007: a complete re-implementation of the GENEPOP software for Windows and Linux. Mol. Ecol. Notes 8: 103-106.

Van Oosterhout C, Hutchinson WF, Wills DPM and Shipley P (2004). Micro-checker: software for identifying and correcting genotyping errors in microsatellite data. Mol. Ecol. Notes 4: 535-538.

Zhan A, Hu J, Hu X, Zhou Z, et al. (2009). Fine-scale population genetic structure of Zhikong scallop (Chlamys farreri): do local marine currents drive geographical differentiation? Mar. Biotechnol. 11: 223-235.

Zhang P, Yang L, Zhang XF and Tang YG (2010). The present status and prospect on exploitation of tuna and squid fishery resources in South China Sea. South China Fish. Sci. 6: 68-74. 